\title{
Sum Power Iterative Waterfilling for Gaussian Vector Broadcast Channels
}

\author{
Sriram Vishwanath*, Wonjong Rhee ${ }^{\dagger}$, Nihar Jindal*, Syed Jafar* and Andrea Goldsmith* \\ "Dept. of Electrical Engineering, $\quad{ }^{\dagger}$ ArrayComm Inc. \\ Stanford, CA $94305 . \quad$ San Jose, CA. \\ \{sriram, njindal, syed, andrea\}@wsl.stanford.edu, wonjong@dsl.stanford.edu
}

\begin{abstract}
We obtain an efficient algorithm for computing the sum capacity of vector broadcast channel. This algorithm utilizes the duality between broadcast and multiple access channels and the Kuhn-Tucker conditions of sum power multiple access channel.
\end{abstract}

\section{INTRODUCTION}

A vector broadcast channel is a one-to-many channel, where the channel between the central transmitter and the receiver $i$ out of $K$ receivers is given by matrix $H_{i}$. Mathematically, such a channel is given by $y_{i}=H_{i} x+n_{i}$, where $y_{i}$ and $n_{i}$ are respectively the received signal vector and the additive Gaussian noise vector at receiver $i$, and $x$ is the transmitted signal vector.

Recently, an achievable region of this channel, known as the dirty paper region, was characterized [1], and the region was shown to achieve sum capacity by many research groups simultaneously (See journal version of [1] and the references therein). The dirty paper characterization in [1] is in terms of transmit covariance matrices $\left\{Q_{i}\right\}_{i=1}^{K}$, where $Q_{i}$ corresponds to user $i$. This characterization, however, turns out to be non-convex in $\left\{Q_{i}\right\}$. In this paper, our aim is to find an efficient algorithm to compute the optimal $\left\{Q_{i}\right\}$ such that the sum capacity of the broadcast channel can be computed.

Simultaneously, a duality result was obtained in [2] showing that the capacity region of the vector multiple access channel (MAC) with sum power constraint on the transmitters is equal to the dirty paper achievable region. Moreover, an explicit transformation connects the optimal transmission scheme for the MAC with the $\left\{Q_{i}\right\}$ above. Thus, we obtain an efficient algorithm to solve the convex dual MAC problem given by

$$
\max _{\left\{S_{i}: \sum_{i=1}^{K} \operatorname{Tr}\left(S_{i}\right) \leq P, S_{i} \geq 0 \forall i\right\}} \log \left|I+\sum_{i=1}^{K} H_{i}^{\dagger} S_{i} H_{i}\right|
$$

and then transform the solution using duality to obtain $\left\{Q_{i}\right\}$. An algorithm to compute the optimal transmit policy for a MAC with per-user power constraints on the transmitters was obtained in [3]. However, this algorithm cannot be directly applied to the dual MAC due to the difference in the power constraint.

\section{THE ALGORITHM}

The iterative algorithm converges to a fixed point which satisfies the Kuhn-Tucker conditions of (1), and hence obtains the solution. Let $S_{i}(l)$ denote the $l^{\prime}$ 'th iteration of $S_{i}$. Then the algorithm can be summarized as follows:

1. Initialize covariance matrices to zero: $S_{i}(0)=0 \quad \forall i$.

2. For iteration $l$ : Generate effective channels $H_{j}^{\text {eff }}=H_{j}(I+$ $\left.\sum_{i \neq j}^{M} H_{i}^{\dagger} S_{i}(l-1) H_{i}\right)^{-1 / 2}$.

${ }^{0}$ This work was supported by a Stanford Graduate Fellowship
3. Treating these effective channels as parallel, non-interfering channels, obtain covariance matrices $M_{i}$ by waterfilling with total power $P$.

$$
\left\{M_{i}(l)\right\}_{i=1}^{K}=\operatorname{argmax} \sum_{i=1}^{K} \log \left|I+\left(H_{i}^{\text {eff }}\right)^{\dagger} A_{i} H_{i}^{\text {eff }}\right|
$$

over the set $A_{i} \geq 0, \sum_{i=1}^{K} \operatorname{Tr}\left(A_{i}\right)=P$

This maximization is equivalent to waterfilling the block diagonal channel with diagonals equal to $H_{j}^{e f f}$.

4. Compute the new $S_{i}(l)=\frac{(K-1) S_{i}(l-1)+M_{i}(l)}{K}$ for all $i$.

5. Return to Step 2 until desired accuracy is reached.

\section{CONVERGENCE AND OPTIMALITY}

Here, we provide an outline for convergence and optimality. First, convergence is considered. Define function $f$ as below:

$$
f\left(S_{1}, S_{2}, \cdots, S_{K}\right)=\log \left|I+\sum_{i=1}^{K} H_{i}^{\dagger} S_{i} H_{i}\right|
$$

Then, the following can be shown.

$$
\begin{aligned}
K f( & \left.S_{1}(l-1), S_{2}(l-1), \cdots, S_{K}(l-1)\right) \\
\leq & f\left(M_{1}(l), S_{2}(l-1), \cdots, S_{K}(l-1)\right) \\
& +f\left(S_{1}(l-1), M_{2}(l), \cdots, S_{K}(l-1)\right) \\
& +\cdots+f\left(S_{1}(l-1), S_{2}(l-1), \cdots, M_{K}(l)\right) \\
\leq & K f\left(\frac{(K-1) S_{1}(l-1)+M_{1}(l)}{K}, \cdots\right. \\
& \left.\ldots, \frac{(K-1) S_{K}(l-1)+M_{K}(l)}{K}\right)
\end{aligned}
$$

The inequality given by Equation (2) is due to the optimality of single-user waterfilling in step 3, and the inequality given by Equation (3) is due to the concavity of $f(\cdot)$ and Jensen's inequality. Jensen's inequality guarantees a strict increase of the function value when any of $S_{i}(l)$ is different from $S_{i}(l-1)$. Therefore, the function value monotonically increases. However, the function value is upper bounded, and we can conclude that the algorithm converges to a fixed point. Note that no loop can exist due to the strict inequality for $S_{i}(l) \neq S_{i}(l-1)$.

Also, $S_{i}$ can be shown to converge to an optimal point. Due to the concavity of the problem, Kuhn-Tucker conditions are necessary and sufficient for optimality. The Kuhn-Tucker conditions can be derived in a similar way as in [3], and can be shown to be satisfied by the limit of $\left\{S_{i}\right\}$ due to steps 2 and 3 of the algorithm.

\section{REFERENCES}

[1] G. Caire and S. Shamai, "On the Achievable Throughput of a Multiantenna Gaussian Broadcast Channel", Proc. IEEE Intl. Symp. Inform. Theory, ISIT 2001, Washington D.C., June 2001. Also submitted to the IEEE Trans. Inform. Theory.

[2] S. Vishwanath, N. Jindal and A. Goldsmith, "Sum Capacity of MultiAntenna Gaussian Broadcast Channels", Proc. IEEE Intl. Conf. Commun., 2001.

[3] W. Yu, W. Rhee, S. Boyd and J. Cioffi, "Iterative Water-filling for Vector Multiple Access Channels", IEEE Intl. Symp. Inform. Theory, (ISIT), 2001. 